Article

\title{
Design of a Pixelated Imaging System for Fast Neutron Sources
}

\author{
John Chatzakis ${ }^{1,2, *}$, Iraklis Rigakis ${ }^{1}$, Syed Hassan ${ }^{2}$, Eugene Laurence Clark ${ }^{2}$, Paul Lee ${ }^{3}$ \\ and Michael Tatarakis 1,2 \\ 1 Department of Electronic Engineering, Hellenic Mediterranean University, 73133 Chania, Crete, Greece; \\ rigakis@chania.teicrete.gr (I.R.); m.tatarakis@chania.teicrete.gr (M.T.) \\ 2 Institute of Plasma Physics and Lasers, Hellenic Mediterranean University, 74100 Rethymno, Crete, Greece; \\ syed.plasma@gmail.com (S.H.); e.clark@chania.teicrete.gr (E.C.) \\ 3 Natural Sciences \& Science Education, National Institute of Education, Nanyang Technological University, \\ Singapore 637616, Singapore; paul.lee@nie.edu.sg \\ * Correspondence: xatzakis@chania.teicrete.gr; Tel.: +30-28210-23002
}

Received: 18 April 2019; Accepted: 21 May 2019; Published: 23 May 2019

\begin{abstract}
Imaging detectors that use X-ray radiation and pulsed neutron sources have increased in sophistication in recent years due to the use of solid-state detectors. A key method for neutron detection is the nuclear activation of materials by neutrons. Neutron activation can generate radionuclides whose decay produces secondary particle emission that can be detected without interference from the X-rays and other prompt radiation sources and offers advantages over neutrons detection using scintillators. In this paper, we present the design of an imaging system for fast neutron sources. The imaging system utilizes a microcontroller network that communicates using a modified SPI protocol. This network communicates with an interface unit and passes an image to a personal computer. A computer program has been developed to reconstruct the image.
\end{abstract}

Keywords: X-rays imaging; fast neutron imaging; solid-state electronics

\section{Introduction}

The detection and imaging of fast neutrons produced in fusion reactions is complicated due to the fact that neutrons are generally measured due to secondary processes as they interact with matter [1,2]. Unlike X-rays, neutrons do not interact directly with electrons. Typically, fast neutrons can be measured using proton recoil techniques, scintillators and neutron induced nuclear reactions. For the latter case, prompt secondary particles can be detected or the decay products of activation can be measured. In some cases, fast neutrons can be moderated to be in an energy regime where the cross section for a nuclear reaction is enhanced so as to increase the sensitivity of the measurement. The detection of neutrons by activation techniques can also suppress the contribution to the measured signal due to electrons and high energy photons. Neutron imaging in fusion experiments [3] typically combines a scintillator screen coupled to an intensified camera or microchannel plate. In this paper we utilize the post-shot activation of beryllium to suppress the contribution to the neutron image due to unwanted ionizing radiations and to formulate a design for a low cost, medium resolution neutron imaging detector.

For the application in this paper we choose a fast neutron reaction that complements the deuterium fusion reaction (2.45 MeV neutron) that is produced by a plasma focus device [4]. Pulsed power devices can also emit $X$-rays as well as a high yield of fast neutrons. X-ray imaging has been in use for decades and is most commonly implemented every day in the diagnosis of the human body's internal structure. X-ray and neutron radiography offer complementary methods for non-destructive testing of objects 
with applications to nondestructive testing, metallurgy, restoration of antiquities [5] and homeland security [6]. The plasma focus, pulsed power device offers an affordable compact source for generating intense point like sources of neutrons and $X$-rays for point projection radiography [7]. The plasma focus can also be configured as a source of fast $2.45 \mathrm{MeV}$ neutrons through the deuterium fusion reaction with many applications including radiography to the calibration of dark matter detectors [8]. The most common method for neutrons imaging is through the use of scintillators that produce light as neutrons or other type of ionizing radiations passing through them. Fast neutron imaging still faces problems [9] when using scintillators due to the fact that scattered low energy neutrons can contribute unwanted signal to the image. The emitted light from a scintillator can be detected using high gain optical sensors such as photomultiplier tubes, intensified cameras and microchannel plates [10]. Scintillators have a disadvantage in that in some applications that they cannot separate X-rays from neutrons [11]. For neutron imaging using pulsed neutron sources, high performance neutron detectors are required in order to discriminate the neutron yield from any accompanying prompt $X$-ray source. There is also the requirement to operate a pulsed neutron device at a high repetition rate so the diagnostic requires a fast readout. To measure the total neutron yield it is now possible to use neutron nuclear activation methods coupled to solid-state detectors that fulfill these requirements [12]. This method detects the particles of the secondary particle emission of the generated radionuclides decay that takes place after the pulsed neutron emission has taken place [13]. The activation is only due to neutron emission so the neutron yield can be discriminated from the prompt X-ray yield by measuring the post-shot activation. Nuclear activation has the advantage in that it can be synchronized with the pulsed neutron source to integrate more than a single pulsed source of radiation, thereby increasing the image contrast. The nuclear decay of activated materials occurs on the order of the half-life of the material. The radionuclide decay releases secondary radiations such as beta particles in the region of several hundred per square centimeter for a moderate fluence of incident neutrons. Wide active area silicon detectors can be used, for example inexpensive pin diodes, to detect this beta emission. The charge produced as the secondary particle passes through the diode can be measured. Low cost pin diodes can be efficiently used today to cover a wide area and provide spatial resolution. This is possible due to the development of low-noise electronic devices and circuits that are capable of operating at room temperature for this purpose. As a result a solid-state dual detector of both X-Rays and fast neutrons is also possible and can be used as a camera for radiography applications. In this paper we present a neutron imaging detectors that uses fast neutron beryllium activation to be used with a plasma focus device. The post-shot activation of the beryllium is measured using low cost photodiodes to determine the subsequent beta emission as a function of space. The detector can also be used for X-ray radiography.

\section{Proposed System Architecture}

The proposed system is consisted of two main parts. One part is to image the prompt X-rays and the other is to measure the fast neutrons by measuring the post-shot activation. The plasma focus can emit both X-rays and a high yield of fast neutrons. X-rays travel at the speed of light, while neutrons travel with speed that is depended of their energy and for fusion neutron sources this is typically a few $10^{7} \mathrm{~m} / \mathrm{s}$. The detector is constructed with a beryllium screen placed at the front of an array of photodiode detectors. These photodiode that are designed as light detectors are capable of measuring electrons and X-rays on the $\mathrm{keV}$ and above range. Each photodiode has an area of $1 \mathrm{~cm}^{2}$ which defines the effective pixel size of the imaging detector. For this reason we envisage this imaging detector as being useful for determining the gross features of large concealed objects. The beryllium screen is transparent to X-rays of sufficient energy and is composed predominantly of ${ }^{9} \mathrm{Be}$. When a fast neutron is incident onto the beryllium screen, a $(n, \alpha)$ reaction occurs. The result of this reaction is ${ }^{6} \mathrm{He}$ and a prompt $\alpha$-particle. The helium- 6 subsequently decays with a half-life of $800 \mathrm{~ms}$ into a dominant beta channel. The beta has an endpoint energy of $3.5 \mathrm{MeV}$ and can be detected from the charge it produces as it pass through the depletion region of a photodiode. The cross section for the neutron 
activation of beryllium peaks at about 0.1 barn for $3 \mathrm{MeV}$ neutrons and so it is ideally suited for the measurement of $2.45 \mathrm{MeV}$ neutrons produced from the D-D reactions in the compact plasma focus. To get an estimate of the minimum sensitivity of the detector, it is necessary to calculate the number of ${ }^{6} \mathrm{He}$ produced per square $\mathrm{cm}$ of the diode area assuming that it is covered in a square $\mathrm{cm}$ of beryllium. For a $2 \mathrm{~mm}$ thick piece of beryllium, there is a requirement of 400 neutrons per $\mathrm{cm}^{2}$ to produce a single ${ }^{6} \mathrm{He}$ and therefore the emission of one beta. The beta could be produced at any time after the neutron irradiation. For good statistics and counting, the neutron irradiation needs to be significantly greater than 400 neutrons $/ \mathrm{cm}^{2}$. For an object placed $10 \mathrm{~cm}$ from the source with the imaging array place directly behind, this suggests the near isotropic neutron emission from the plasma focus device needs to be in excess of $10^{5}$ neutrons per shot. To achieve a sufficient neutron yield the plasma focus can be operated at a high repetition rate of $1 \mathrm{~Hz}$ to build up sufficient counts in the detector.

At the moment of the radiation emission from the plasma focus device, the photodiodes, that are used as detectors, are connected in an X-rays detector Analog Front End (AFE). This Analog Front End contains the necessary circuitry and the Sample and Hold $(\mathrm{S} / \mathrm{H})$ units to keep the image that is produced from the $\mathrm{X}$-rays. Instantly after the emission and the $\mathrm{X}$-rays image holding to $\mathrm{S} / \mathrm{H}$ units of the AFE, the photodiodes are switched to the Charge Amplifiers that are the input of the Neutron Imaging Unit. This unit counts the beta particle pulses for more than 2.5 seconds after the activation has occurred. Meanwhile, the X-ray Imaging Unit reads the sample and hold units and passes the $\mathrm{X}$-rays Image to a Personal Computer (PC) that will show the image using a Computer Program that was developed for this purpose. The Neutron Imaging Unit detects the betas produced from the neutrons on the beryllium screen. This unit is synchronized with the source to integrate several emission pulses for an acceptable signal-to-noise ratio when the yield per shot is low [3].

After the end of the pulse reading of the Neutrons Imaging Unit the number of pulses of each pixel is interpreted in an intensity value and this way a neutron image is created. This image is also passed to the appropriate computer program and is shown on the PC screen. The pixel processing block diagram of the proposed system is shown in Figure 1.

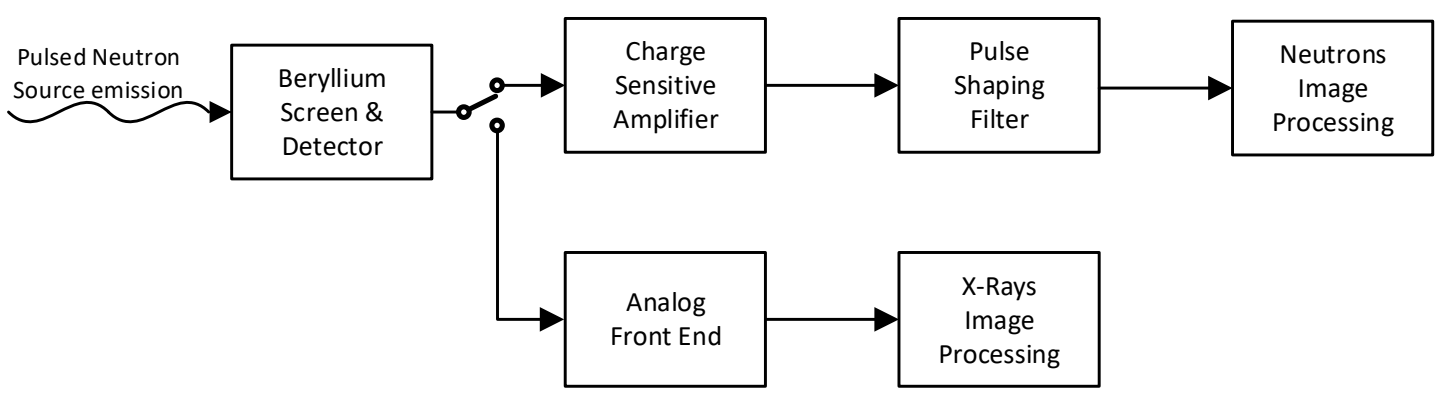

Figure 1. The proposed charge amplifier circuit.

The detector is a p-i-n (p-type semiconductor/n-type semiconductor with wide intrinsic semiconductor in between) photodiode that is switched between the X-ray Image Processing Unit and the Neutron Image Processing Unit. Initially it is connected to the X-ray detector AFE that enables the $\mathrm{S} / \mathrm{H}$ units exactly before the plasma focus is ready for emission. After the emission the X-ray image information is almost instantly stored on the AFE S/H units. The p-i-n photodiode is then switched to the charge sensitive amplifier. The neutrons arrive at a time that depends on their energy and the distance of the imaging device from the neutron source. The neutrons interact with the beryllium Screen that is activated with a half-life of $800 \mathrm{~ms}$. After $2.5 \mathrm{sec}$ almost all the beta particles that are produced from the activation will pass through the p-i-n photodiode. Each time a beta particle pass through the photodiodes depletion region, charge is produced and stored to Charge Amplifier's capacitor, producing a pulse on its output. This pulse is shaped with the use of a Pulse Shaping Filter. In this way, the pulse to noise ratio is increased and the pulse becomes detectable. 


\section{Experimental Results}

To test the ability of the photodiodes to count beta particles, a Sr-90 beta emitting source was used so as to simulate the effect of beta particles emitted from the beryllium screen when irradiated with neutrons. For each beta that pass through the photodiode a pulse will appear at the output of the Pulse Shaping Filter, as shown in Figure 2, with an amplitude that is dependent on the position and the angle that it passed through the photodiode. This pulse must be discriminated and counted before passing to the Neutron Image Processing Unit.

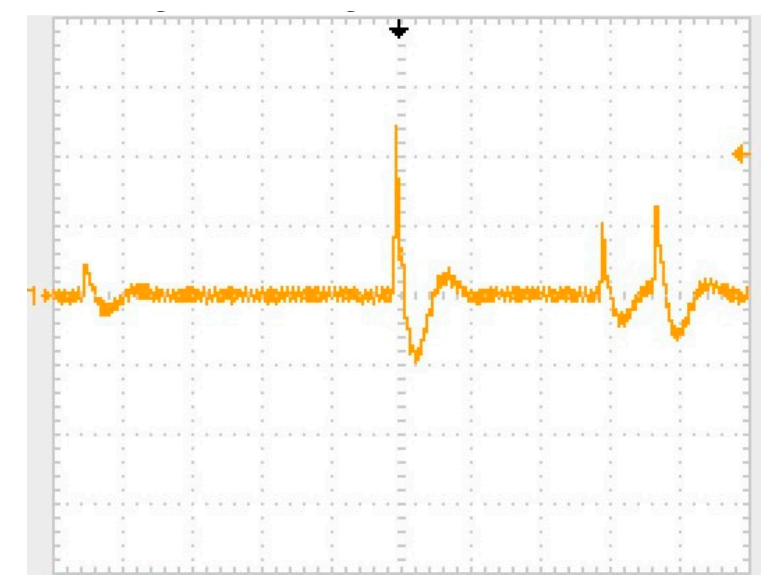

Figure 2. Oscilloscope image of several pulses in the output of the pulse shaping filter circuit. Vertical scale is $2 \mathrm{~V} /$ division, horizontal scale is 250 microseconds/division.

Each photodiode in the imaging array formulates a pixel in the imaging device and thus requires its own independent circuitry for pulse counting. A low cost ultra-low power microcontroller (MCU) is capable of handling 2 pixels. So the basic unit for the neutrons image processing consists of two photodiodes and their respective analog circuits (charge amplifiers, pulse shaping filters and voltage comparators) and one microcontroller. Each microcontroller has also an input that is fed with signal from a dedicated photodiode that measures the time of arrival of the X-ray pulse. The time difference of the first beta particle pulse of the whole sensor and the arrival of the $X$-rays can give a good estimation of how far away the neutron source is for $2.45 \mathrm{MeV}$ neutrons. The block diagram of the basic unit for the neutrons image processing is shown in Figure 3.

The microcontroller counts the pulses from the two comparators outputs. In order to avoid false counts the threshold voltage of each comparator must be above the noise level of the pulse shaping filter output. For this reason, before the system starts counting, the microcontroller reads continuously the analog output of each pulse shaping filter, calculates the maximum noise value $V_{n}$ and sets the voltage comparator thresholds $20 \%$ above $V_{n}$. The pulse shaping filters have a central frequency of about $15 \mathrm{kHz}$ and the sampling rate is at least an order of magnitude higher. The threshold voltage of each comparator, produced by one internal PWM unit of the microcontroller, feeds the comparator's threshold input through a passive low-pass filter. A close view of an experimental basic unit for the neutrons image processing is shown in Figure 4. Shielding is significant close to the photodiodes, as the signals are very low and noise can affect strongly the operation of the circuit. 


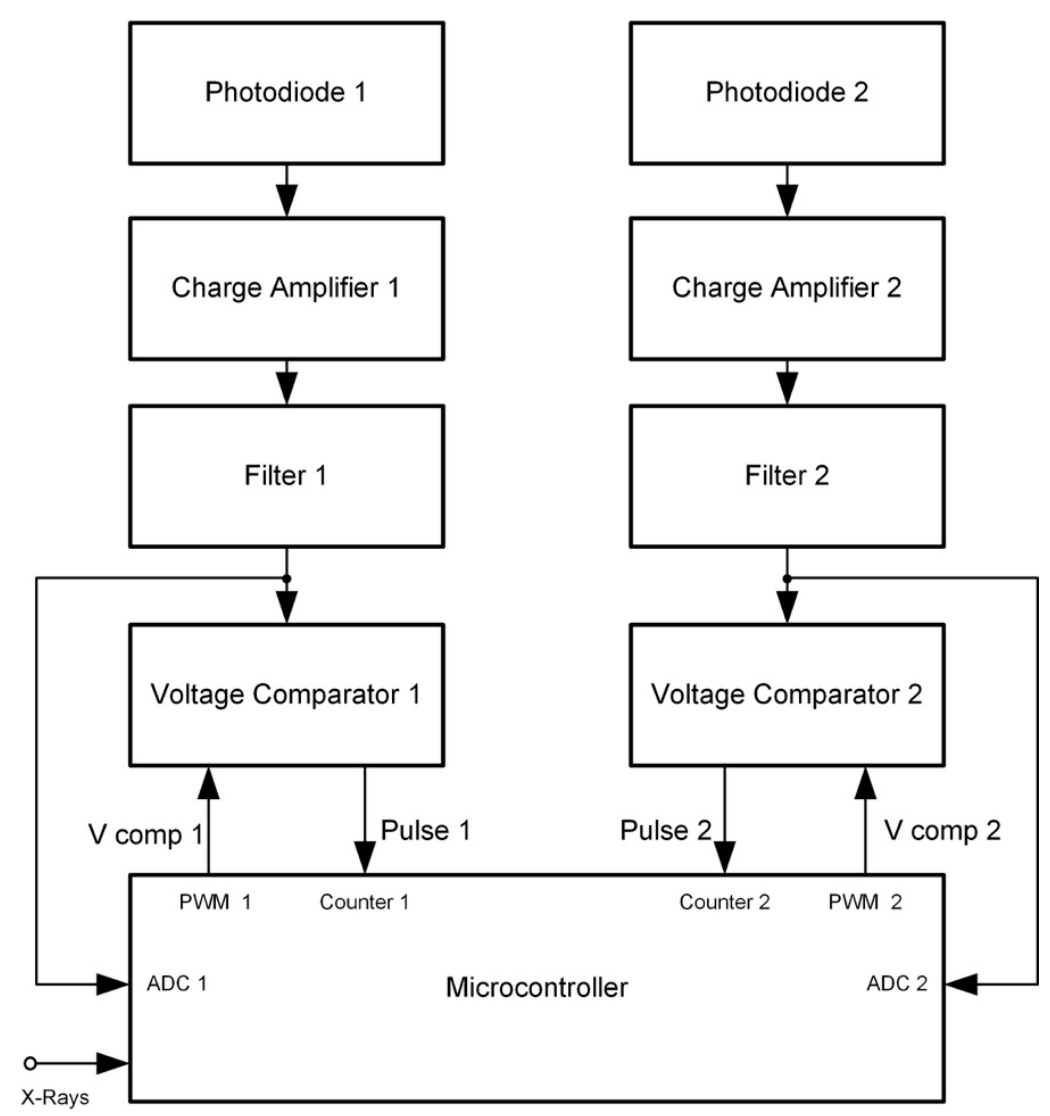

Figure 3. Block diagram of the basic unit for the neutrons image processing.

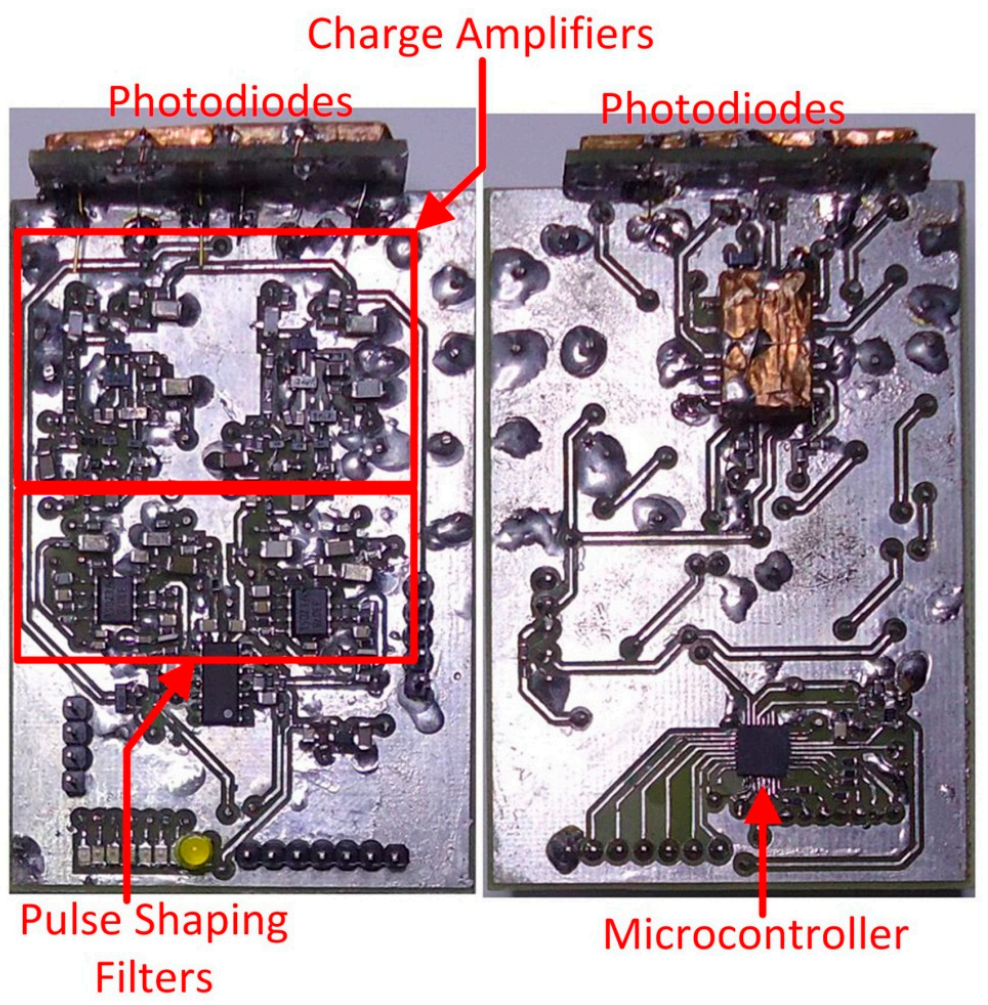

Figure 4. The basic unit for the neutrons image processing. 
The microcontrollers of the basic units for the neutrons image processing allow local pixel processing and give smart capabilities to the image sensor. All of them are slaves on a microcontroller network that communicates using a high speed modified bidirectional SPI Bus and a specific protocol that was developed for this purpose. A dedicated master microcontroller is controlling the network communications and is capable of giving orders to all or each one of the slave microcontrollers. The master microcontroller communicates also with a PC through a RS232 to Universal Serial Bus (USB) port and collects the data from both $\mathrm{X}$-rays and neutrons imaging units. In addition, two more signals are used, the reset (RST), in order to reset the slave microcontrollers, and the trigger that initializes the image sensor. This trigger can be used to synchronize the detector with the plasma focus radiation emission when more than one shots are required to form an image [14]. When the slave receives the trigger pulse, it connects the photodiodes to the $\mathrm{X}$-rays AFE. Once the $\mathrm{X}$-ray image has been captured, it connects the photodiodes to the charge amplifiers in order to start the counting of the pulses. The communications block diagram is shown in Figure 5.

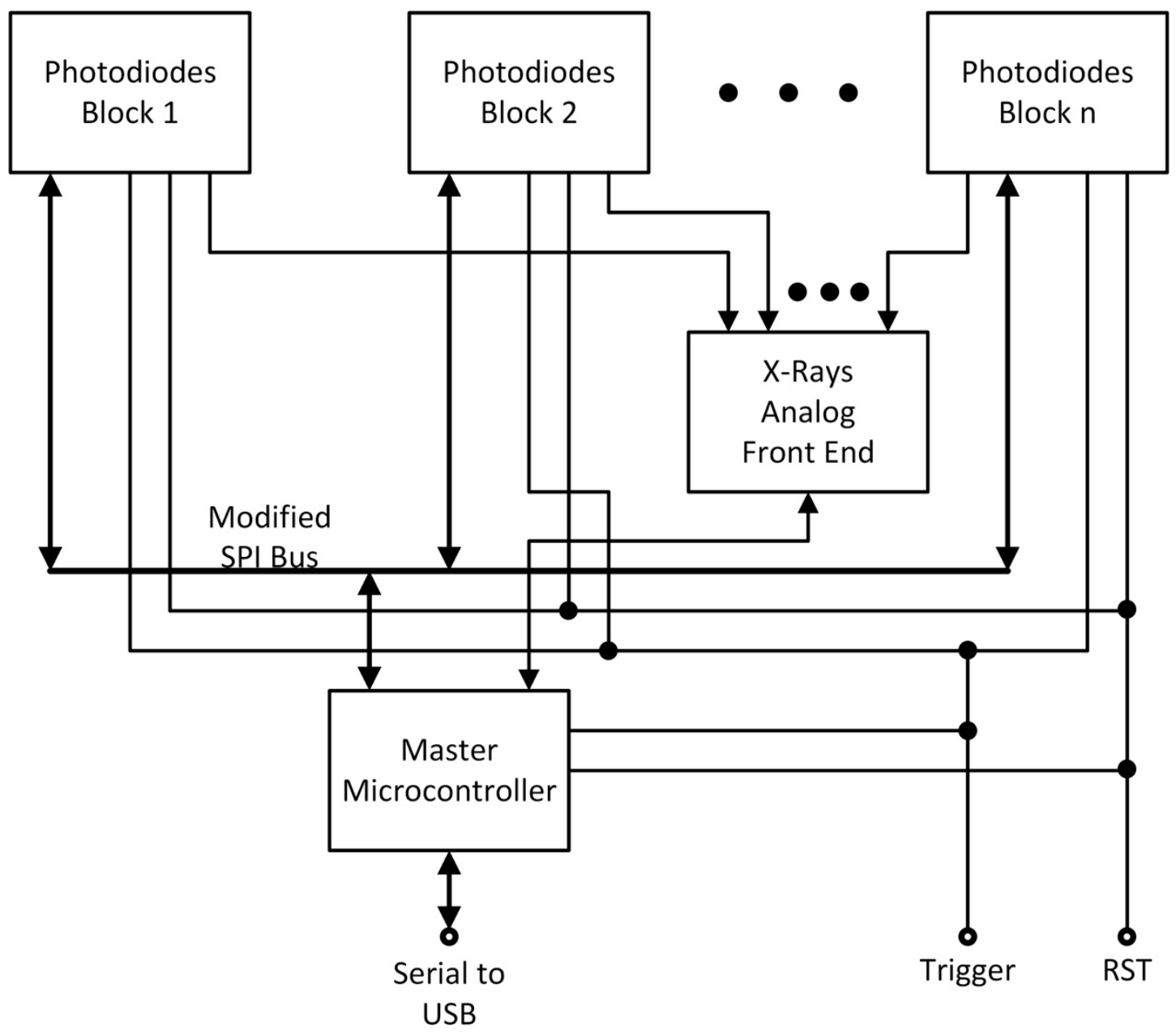

Figure 5. Block diagram of the image sensor communications.

The image sensor through the Master Microcontroller communicates with software developed for this application. The graphical user interface that has been developed for experimental use is capable of displaying images of both the X-ray AFE and neutron comparators.

\section{Conclusions}

The design of a solid-state imaging system capable of displaying images from both X-rays and fast neutrons that are emitted from a plasma focus device is described in this paper. The neutron detection 
is based on nuclear activation caused by neutrons, and the detection of the secondary particle emission of their decay. The proposed imaging system utilizes a microcontroller's network that adds smart capabilities to it. A computer program is capable of communicating with the imaging system, in order to control it and show and process the produced radiographic images. An experimental prototype has been constructed and evaluated in the laboratory using X-rays from pulsed X-ray sources and by using a Sr-90 source that emits betas with sufficient energy to propagate into the active layer of the p-i-n photodiodes. We propose to test the diagnostic with a $2.45 \mathrm{MeV}$ neutrons from a plasma focus device in the near future.

Author Contributions: Methodology, J.C., I.R., S.H., E.L.C., P.L. and M.T.; Project administration, J.C.

Funding: This research received no external funding.

Conflicts of Interest: The authors declare no conflict of interest.

\section{References}

1. Tsoulfanidis, N. Measurement and Detection of Radiation; Taylor \& Francis: Washington, DC, USA, 1995; pp. 467-501. ISBN 978-1-56032-317-4.

2. Reilly, D.; Ensslin, N.; Smith, H., Jr.; Kreiner, S. Passive Nondestructive Assay of Nuclear Materials; Nuclear Regulatory Commission: Washington, DC, USA, 1991; p. 379. ISBN 0-16-032724-5.

3. Merrill, F.E.; Bower, D.; Buckles, R.; Clark, D.D.; Danly, C.R.; Drury, O.B.; Dzenitis, J.M.; Fatherley, V.E.; Fittinghoff, D.N.; Gallegos, R.; et al. The neutron imaging diagnostic at NIF. Rev. Sci. Instrum. 2012, 83. [CrossRef] [PubMed]

4. Skoulakis, A.; Androulakis, G.C.; Clark, E.L.; Hassan, S.M.; Lee, P.; Chatzakis, J.; Bakarezos, M.; Dimitriou, V.; Petridis, C.; Papadogiannis, N.A.; et al. A portable pulsed neutron generator. Int. J. Mod. Phys. Conf. Ser. 2014, 27, 1460127. [CrossRef]

5. Lehmann, E.; Mannes, D.; Kaestner, A.; Grünzweig, C. Recent Applications of Neutron Imaging. Method. Phys. Proced. 2017, 88, 5. [CrossRef]

6. Gaukler, G.M.; Li, C.; Cannaday, R.; Chirayath, S.S.; Ding, Y. Detecting nuclear materials smuggling: Using radiography to improve container inspection policies. Ann. Oper. Res. 2011, 187, 65-87. [CrossRef]

7. Beg, F.N.; Krushelnick, K.; Lichtsteiner, P.; Meakins, A.; Kennedy, A.; Kajumba, N.; Burt, G.; Dangor, A.E. Table-top X-pinch for x-ray radiography. Appl. Phys. Lett. 2003, 82, 4602. [CrossRef]

8. Beg, F.N.; Krushelnick, K.; Gower, C.; Torn, S.; Dangor, A.E.; Howard, A.; Sumner, T.; Bewick, A.; Lebedenko, V.; Dawson, J.; et al. Table-top neutron source for characterization and calibration of dark matter detectors. Appl. Phys. Lett. 2002, 80, 3009-3011. [CrossRef]

9. Chandra, R.; Davatz, G.; Gendotti, U.; Howard, A. Gamma-Insensitive Fast Neutron Detector with Spectral Source Identification Potential. Available online: https://www.hsdl.org/?view\&did=7668 (accessed on 22 May 2019).

10. Nagarkar, V.V.; Ovechkina, E.E.; Bhandari, H.B.; Soundara-Pandian, L.; More, M.J.; Riedel, R.A.; Miller, S.R. New structured scintillators for neutron radiography. Phys. Proced. 2015, 69, 161-168. [CrossRef]

11. Caruso, A.N. The physics of solid-state neutron detector materials and geometries. J. Phys. Condens. Matter 2010, 22, 443201. [CrossRef] [PubMed]

12. Chatzakis, J.; Rigakis, I.; Hassan, S.M.; Clark, E.L.; Lee, P. Detection of pulsed neutrons with solid-state electronics. Int. J. Mod. Phys. Conf. Ser. 2016, 44, 1660229. [CrossRef]

13. Fan, R.; Ma, J.; Ai, J. A silver activation detector used in pulsed neutron measurement. Nucl. Electron. Detect. Technol. 2008, 28, 875-877.

14. Talebitaher, A.; Springham, S.V.; Rawat, R.S.; Lee, P. Nuclear Instruments and Methods in Physics Research Section A: Accelerators, Spectrometers, Detectors and Associated Equipment. Nucl. Instrum. Method. Phys. Res. 2011, 659, 361-367. [CrossRef]

(C) 2019 by the authors. Licensee MDPI, Basel, Switzerland. This article is an open access article distributed under the terms and conditions of the Creative Commons Attribution (CC BY) license (http://creativecommons.org/licenses/by/4.0/). 